\title{
Article \\ Trends in In-Hospital Cardiopulmonary Resuscitation from 2010 through 2019: A Nationwide Cohort Study in South Korea
}

\author{
Tak Kyu Oh ${ }^{1,2}$, You Hwan Jo ${ }^{3}$ and In-Ae Song ${ }^{1, *}$
}

check for updates

Citation: Oh, T.K.; Jo, Y.H.; Song, I.-A. Trends in In-Hospital

Cardiopulmonary Resuscitation from 2010 through 2019: A Nationwide

Cohort Study in South Korea. J. Pers. Med. 2022, 12, 377. https:// doi.org/10.3390/jpm12030377

Academic Editor: Hyesun Gwak

Received: 20 January 2022

Accepted: 28 February 2022

Published: 1 March 2022

Publisher's Note: MDPI stays neutral with regard to jurisdictional claims in published maps and institutional affiliations.

Copyright: (C) 2022 by the authors. Licensee MDPI, Basel, Switzerland. This article is an open access article distributed under the terms and conditions of the Creative Commons Attribution (CC BY) license (https:// creativecommons.org/licenses/by/ $4.0 /)$.
1 Department of Anesthesiology and Pain Medicine, Seoul National University Bundang Hospital, Seongnam 13620, Korea; airohtak@hotmail.com

2 Department of Anesthesiology and Pain Medicine, College of Medicine, Seoul National University, Seoul 04551, Korea

3 Department of Emergency Medicine, Seoul National University Bundang Hospital, Seongnam 13620, Korea; drakejo@snubh.org

* Correspondence: songoficu@outlook.kr

\begin{abstract}
We aimed to examine recent trends in in-hospital cardiopulmonary resuscitation in South Korea from 2010 to 2019. A population-based sample of all adult patients who experienced inhospital cardiopulmonary resuscitation between 1 January 2010 and 31 December 2019, was included. In all, 298,676 patients who received in-hospital cardiopulmonary resuscitation were included in the survival analysis. In 2010, 60.7 per 100,000 adults experienced in-hospital cardiopulmonary resuscitation. A similar rate was observed until 2015. The rate increased to 83.5 per 100,000 adults in 2016 and gradually increased to 92.1 per 100,000 adults in 2019. Among all patients, 78,783 (26.2\%) were discharged alive after in-hospital cardiopulmonary resuscitation. The 6-month and 1-year survival rates were $9.8 \%$ and $8.7 \%$, respectively. In 2010, the mean total cost of hospitalization was USD 5822.80 (United States Dollar) (standard deviation; SD: USD 7493.4), which increased to USD 7886.20 (SD: USD 13,071.6) in 2019. The rate of in-hospital cardiopulmonary resuscitation and cost of care have significantly increased since 2010, while the 6-month and 1-year rates of survival post in-hospital resuscitation remain low.
\end{abstract}

Keywords: cardiopulmonary resuscitation; critical care; heart arrest; hospitals; resuscitation

\section{Introduction}

In-hospital cardiac arrest (IHCA) is an acute and critical event that can cause death in any hospitalized patient [1]. The occurrence of IHCA is common in United States hospitals, with a survival rate as low as approximately $20 \%$ [2]. In South Korea, the prevalence of IHCA was 2.46 per 1000 admissions in 2009, according to the National Representative Patient Sample [3].

Cardiopulmonary resuscitation (CPR) is a life-saving procedure that is required for patients who have a cardiac arrest event, and in-hospital CPR (ICPR) should be performed for patients with IHCA [4]. In the United States, the incidence of ICPR has increased, and the overall survival rate was 30.4\% from 2007 to 2012 among non-elderly (18-64 years) patients [5]. Another epidemiologic study reported that, in the United States, $18.3 \%$ of elderly patients ( $\geq 65$ years) survived to discharge after ICPR. In South Korea, according to the National Representative Patient Sample database, there were 5919 ICPR cases from 2003 to 2013, and the live discharge rate was 11.7\% [6]. However, there have been no detailed reports on recent trends regarding prevalence, mortality, factors associated with hospital mortality in South Korea, clinical characteristics, or associated costs for patients who receive ICPR.

Therefore, we aimed to examine recent trends in ICPR in South Korea from 2010 to 2019 using the National Health Insurance Database. Given the increase in the aging population 
in South Korea, the frequency of ICPR due to IHCA is likely to increase. However, hospital mortality rates after ICPR may have improved due to advances in critical care medicine.

\section{Materials and Methods}

\subsection{Study Design, Setting, and Ethical Concerns}

For this nationwide, population-based cohort study, we followed the "Strengthening of the Reporting of Observational Studies in Epidemiology" guidelines [7]. The study protocol was approved by the institutional review board (IRB; X-2011-651-901), and the National Health Insurance Service (NHIS) permitted data sharing after approval of the study protocol (NHIS-2021-1-266). The requirement for informed consent was waived by the IRB because anonymized data were used in this study.

\subsection{Data Source}

The NHIS database was used for this study. As the sole public insurance database system in South Korea, it contains information regarding all disease diagnoses and medication prescriptions and/or procedures. These registrations enable patients to receive financial support from the government for treatment expenses. The International Statistical Classification of Disease and Related Health Problems, 10th Revision (ICD-10) codes were used to diagnose diseases.

\subsection{Study Population}

We initially screened patients who underwent CPR between 1 January 2010 and 31 December 2019. Next, we excluded cases of CPR for out-of-hospital cardiac arrest, and all ICPR cases were selected for this study. For any patient, all cases of ICPR in a day were counted as one ICPR case. For example, if a patient received ICPR four times on a certain day during the study period, it was considered as one ICPR case. If a patient received ICPR two or more times on different days during the study period, only the first ICPR case at the earliest date was included in this study. Pediatric patients were excluded from the analysis. The accurate death dates for all patients included in the study population were extracted and collected until 30 April 2021.

\subsection{Collected Information}

Age and sex were collected as the physical information. To reflect the socioeconomic statuses of the patients included in the study population, employment status, residence, and household income level at ICPR were collected. Self-employed patients were not considered as employed, and residence data, such as residency in urban areas (Seoul and other metropolitan cities) or rural areas, were collected at hospital admission based on ZIP codes. The NHIS collects information regarding household income levels to determine insurance premiums for patients, and the data were divided into four groups using the quartile ratio.

The main diagnoses at ICPR were collected and divided into four groups, according to ICD-10 codes: cardiovascular disease (I00-I99), respiratory disease (J00-J99), cancer (C00-D49), and others. The main diagnosis at ICPR was determined by NHIS after hospital discharge or death as the disease that required the greatest treatment or examination during the patient's hospitalization. The admitting departments at ICPR were identified and classified into internal medicine (IM) and non-IM groups. The duration of ICPR was classified into one of five groups: $<15 \mathrm{~min}, 15-30 \mathrm{~min}, 30-45 \mathrm{~min}, 45-60 \mathrm{~min}$, and $>60 \mathrm{~min}$. The total length of hospital stay (day), and total hospitalization cost (United States Dollar, USD), were collected. The hospitals where the ICPRs were performed during the study period were classified into three groups: tertiary general hospital, general hospital, and other hospital. In addition, hospitals were divided into two groups, according to the total number of hospital beds, including those in intensive care units: $<1000$ beds and $\geq 1000$ beds. The results of treatment at ICPR were collected and classified into four groups: discharge and same hospital follow-up, transfer to a long-term facility care center, death 
during hospitalization after ICPR, discharge, and other outpatient clinic follow-up. Lastly, to reflect the comorbid status of all patients, the Charlson comorbidity index (CCI) score was calculated using ICD-10 codes in the NHIS database, which were registered no more than 1 year before ICPR, as shown in Table S1.

\subsection{Study Outcomes (Trend of ICPR in South Korea)}

First, we examined the prevalence of ICPR between 2010 and 2019. The prevalence of ICPR was calculated as the total number of annual ICPR cases divided by the total adult population in that year. The total population was obtained from the Statistics Korea database (http:/ / kostat.go.kr/portal/eng/index.action, accessed on 7 September 2021). Second, the in-hospital, 6-month, and 1-year mortality rates from 2010 to 2019 were examined. Third, the trend in total costs of hospitalization at ICPR was examined. Fourth, the main diagnosis and total duration of ICPR were examined. Fifth, the factors associated with live discharge after ICPR were examined.

\subsection{Statistical Analysis}

The clinicopathological characteristics of the study population were presented as mean values with standard deviation (SD) for continuous variables and numbers with percentages for categorical variables. We constructed a multivariable logistic regression model for the live discharge rate for patients who experienced ICPR. All covariates were included in the multivariable model, and the results are presented as adjusted odds ratios (aORs) with 95\% confidence intervals (CIs). The goodness-of-fit in the multivariable model was confirmed using the Hosmer-Lemeshow test, and there was no multicollinearity issue between variables with criteria of variance inflation factors <2.0. All statistical analyses were performed using SPSS software (IBM SPSS Statistics for Windows, Version 25.0, IBM Corp., Armonk, NY, USA), and statistical significance was set at $p<0.05$.

\section{Results}

\subsection{Study Population for Survival Analysis}

Figure S1 shows the study population selection process. From 1 January 2010 to 31 December 2019, there were a total of 478,836 CPR cases in South Korea. After the exclusion of 140,046 cases of CPR due to out-of-hospital cardiac arrest, 338,970 ICPR cases were initially screened. Next, 31,122 cases in which a patient received ICPR on two or more days during the study period and 8992 pediatric cases (patients under 18 years of age) were excluded from the final analysis. Finally, 298,676 patients were included in the survival analysis. Among them, 78,783 (26.2\%) were discharged alive after ICPR, and the 6-month and 1-year survival rates were $9.8 \%(29,303 / 298,676)$ and $8.7 \%(25,850 / 298,676)$, respectively. The clinicopathological characteristics of the study population are shown in Table 1 . The mean age was 70.0 years old (SD: 15.2 years old), and the proportion of male patients was $60.4 \%(180,389 / 298,676)$.

\subsection{Trends of ICPR}

Figure 1 and Table S2 show the prevalence of ICPR in South Korea among the adult population from 2010 to 2019. In 2010, 60.7 per 100,000 adults experienced ICPR, and a similar rate was observed until 2015. However, the rate increased to 83.5 per 100,000 adults in 2016 and gradually increased to 92.1 per 100,000 adults in 2019. Figure 2 and Table S3 show mortality rates after ICPR from 2010 to 2019. In-hospital, 6-month, and 1-year mortality rates were $73.1 \%(17,894 / 24,486), 90.5 \%(22,160 / 24,486)$, and $91.7 \%(22,449 / 24,486)$, respectively, in 2010. Rates were similar for 10 years through 2019; thus, the in-hospital, 6-month, and 1-year mortality rates were $74.4 \%(30,080 / 40,433), 91.0 \%(36,806 / 40,433)$, and $92.0 \%(37,194 / 40,433)$, respectively, in 2019. Figure 3 and Table S4 show the total cost of hospitalization at ICPR from 2010 to 2019. In 2010, the mean value of the total cost of hospitalization was USD 5822.80 (SD: USD 7493.40), which increased to USD 7886.20 (SD: USD 13,071.60) in 2019. 
Table 1. Clinicopathological characteristics of the study population.

\begin{tabular}{|c|c|}
\hline Variable & Mean (SD) or N (\%) \\
\hline Age & $70.0(15.2)$ \\
\hline Sex, male & $180,389(60.4)$ \\
\hline Have a job at ICPR & $153,053(51.2)$ \\
\hline \multicolumn{2}{|l|}{ Residence at ICPR } \\
\hline Urban area & $129,057(43.2)$ \\
\hline Rural area & $169,619(56.8)$ \\
\hline \multicolumn{2}{|l|}{ Household income level at ICPR } \\
\hline Q1 & $93,778(31.4)$ \\
\hline Q2 & $46,226(15.5)$ \\
\hline Q3 & $58,628(19.6)$ \\
\hline $\mathrm{Q} 4$ & $94,424(31.6)$ \\
\hline Unknown & $5620(1.9)$ \\
\hline \multicolumn{2}{|l|}{ Main diagnosis at ICPR } \\
\hline Cardiovascular disease & $115,159(38.6)$ \\
\hline Respiratory disease & $41,959(14.0)$ \\
\hline Cancer & $33,498(11.2)$ \\
\hline Other & $108,060(36.2)$ \\
\hline \multicolumn{2}{|l|}{ Admitting department } \\
\hline $\mathrm{IM}^{\mathrm{I}}$ & $166,779(55.8)$ \\
\hline Non-IM & $131,897(44.2)$ \\
\hline \multicolumn{2}{|l|}{ Duration of ICPR } \\
\hline$<15 \mathrm{~min}$ & $128,869(43.1)$ \\
\hline $15-30$ & $86,534(29.0)$ \\
\hline $30-45$ & $40,848(13.7)$ \\
\hline $45-60$ & $19,227(6.4)$ \\
\hline$>60 \mathrm{~min}$ & $16,402(5.5)$ \\
\hline LOS at ICPR & $9.9(13.1)$ \\
\hline Total cost for hospitalization at ICPR, USD & $6753.5(10,295.1)$ \\
\hline Insurance coverage & $6030.2(9490.5)$ \\
\hline CCI & $6.0(3.9)$ \\
\hline \multicolumn{2}{|l|}{ Type of hospital } \\
\hline Tertiary general hospital & $112,290(37.6)$ \\
\hline General hospital & $153,320(51.3)$ \\
\hline Other hospital & $33,066(11.1)$ \\
\hline \multicolumn{2}{|l|}{ Total hospital bed number } \\
\hline$<1000$ & $253,184(84.8)$ \\
\hline$\geq 1000$ & $45,492(15.2)$ \\
\hline \multicolumn{2}{|l|}{ Result of treatment } \\
\hline Discharge and same hospital follow up & $25,617(8.6)$ \\
\hline Transfer to long-term facility care center & $8396(2.8)$ \\
\hline Death within hospitalization after ICPR & $220,493(73.8)$ \\
\hline Discharge and other outpatient clinic follow-up & $44,170(14.8)$ \\
\hline \multicolumn{2}{|l|}{ Year of ICPR } \\
\hline 2010 & $24,486(8.2)$ \\
\hline 2011 & $24,169(8.1)$ \\
\hline 2012 & $24,606(8.2)$ \\
\hline 2013 & $24,092(8.1)$ \\
\hline 2014 & $23,696(7.9)$ \\
\hline 2015 & $23,811(8.0)$ \\
\hline 2016 & $35,880(12.0)$ \\
\hline 2017 & $35,894(12.0)$ \\
\hline 2018 & 41,609 (13.9) \\
\hline 2019 & $40,433(13.5)$ \\
\hline
\end{tabular}

SD, standard deviation; ICPR, in-hospital cardiopulmonary resuscitation; IM, internal medicine; USD, United States Dollar; LOS, length of hospital stays; CCI, Charlson comorbidity index. 


\section{Prevalence of ICPR}

120

100

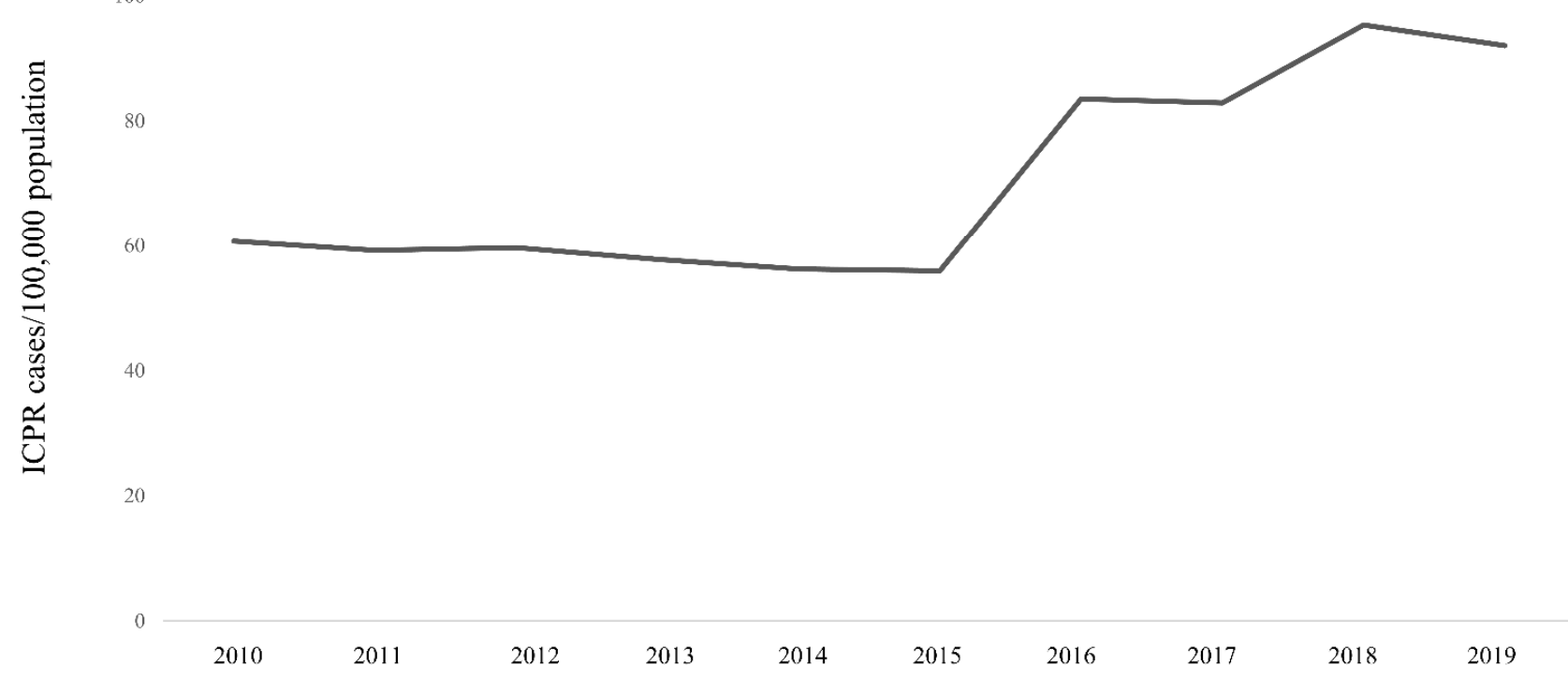

Figure 1. Prevalence of ICPR in South Korea among adult population from 2010 to 2019. ICPR, in-hospital cardiopulmonary resuscitation.

Mortality rates after ICPR

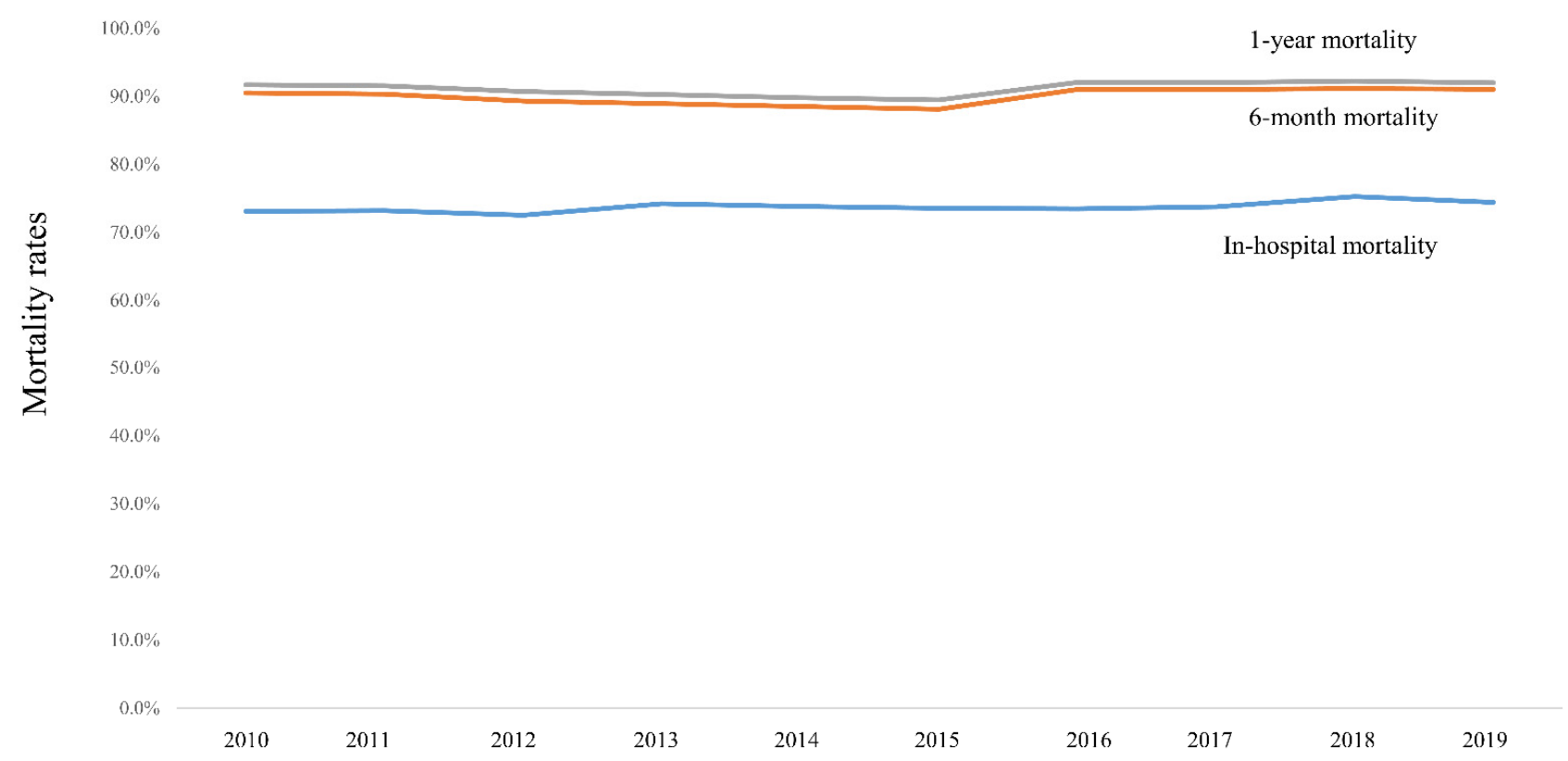

Figure 2. Mortality rates after ICPR from 2010 to 2019. ICPR, in-hospital cardiopulmonary resuscitation. 


\section{Total cost of hospitalization at ICPR}

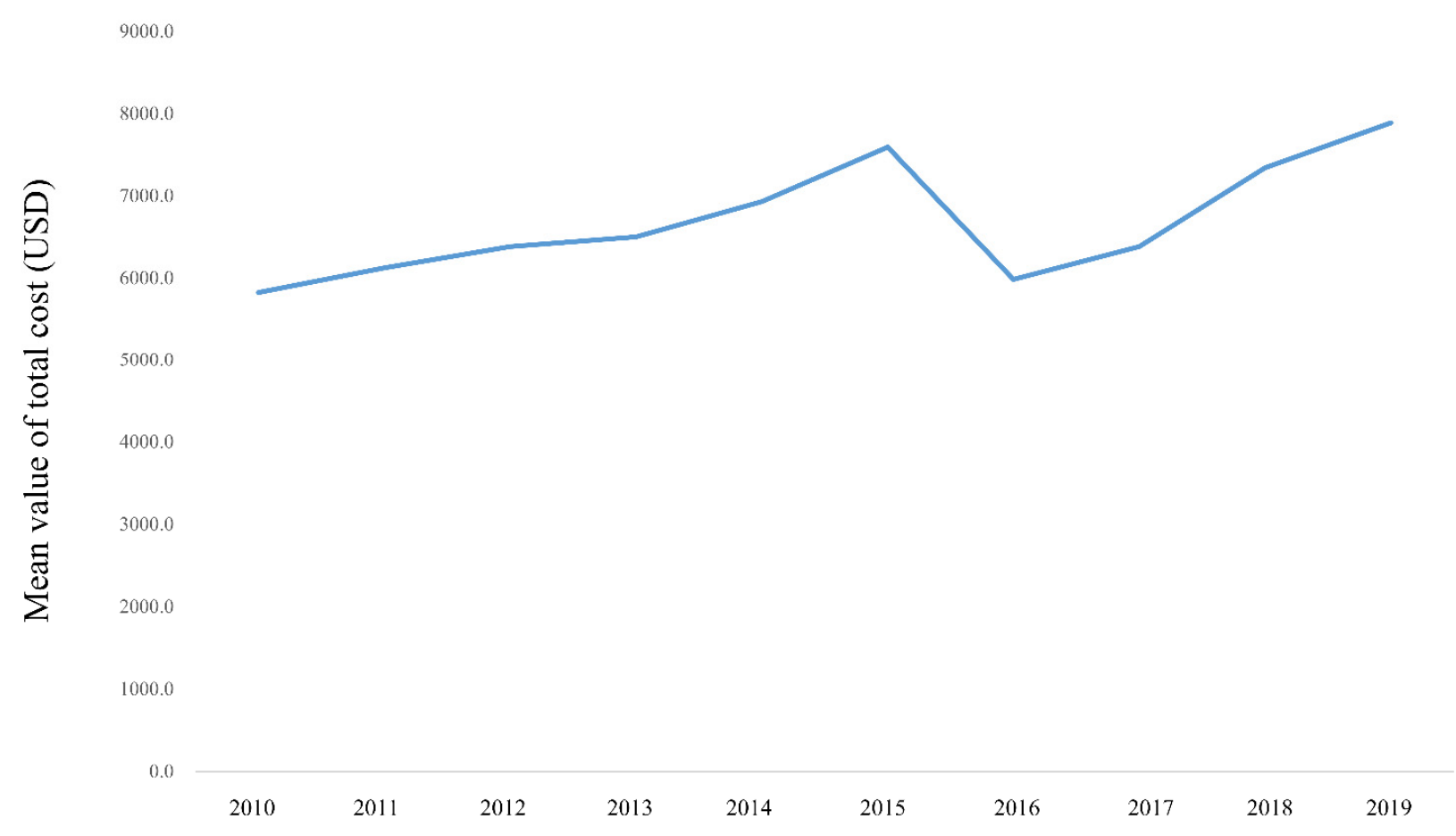

Figure 3. Total cost of hospitalization at ICPR from 2010 to 2019. ICPR, in-hospital cardiopulmonary resuscitation.

Figure S2 and Table S5 show the trends in the main diagnosis at ICPR from 2010 to 2019. The proportion of cardiovascular disease as the main diagnosis in 2010 was $29.3 \%$ $(7174 / 24,486)$ and increased to $44.4 \%(18,472 / 40,433)$ in 2019 . The proportion of respiratory disease as the main diagnosis in 2010 was $16.2 \%(3614 / 24,486)$ and decreased to $11.2 \%$ $(4516 / 40,433)$ in 2019. The proportion of cancer as the main diagnosis in 2010 was $14.8 \%$ $(3614 / 24,486)$ and decreased to $9.0 \%(3627 / 40,433)$ in 2019. Figure S3 and Table S6 show the trends in the duration of ICPR from 2010 to 2019.

\subsection{Associated Factors for Discharge Alive}

Table 2 shows the results of the multivariable logistic regression model for live discharge after ICPR. Older age (aOR: $0.98,95 \%$ CI: $0.98,0.99 ; p<0.001)$ and residency in rural areas (vs. urban areas) (aOR, $0.91 ; 95 \%$ CI: 0.90, 0.93; $p<0.001$ ) were associated with lower odds of live discharge. Compared with cardiovascular disease as the main diagnosis, respiratory disease (aOR: $0.72,95 \%$ CI: 0.70, $0.74 ; p<0.001$ ), cancer (aOR: 0.38, 95\% CI: 0.37, $0.40 ; p<0.001)$, and other main diagnoses (aOR, $0.70 ; 95 \% \mathrm{CI}, 0.68,0.71 ; p<0.001)$ were associated with lower odds of live discharge. In addition, compared with $<15$ min duration of ICPR, $15-30 \mathrm{~min}$ (aOR: $0.42,95 \%$ CI: $0.41,0.43 ; p<0.001$ ), 30-45 $\mathrm{min}$ (aOR: $0.29,95 \%$ CI: $0.28,0.30 ; p<0.001$ ), $45-60 \mathrm{~min}$ (aOR: $0.26,95 \%$ CI: $0.24,0.26 ; p<0.001$ ), and $>60 \mathrm{~min}$ (aOR: $0.25,95 \%$ CI: $0.24,0.26 ; p<0.001$ ) were associated with lower odds of live discharge.

Compared with unemployed patients, employed patients (aOR: 1.05, 95\% CI: 1.03, $1.07 ; p<0.001)$ had higher odds of live discharge. Compared with the first quartile (Q1) of household income level, Q2 (aOR: 1.06, 95\% CI: 1.03, 1.12; $p<0.001$ ), Q3 (aOR: 1.09, 95\% CI: $1.06,1.12 ; p<0.001$ ), and Q4 (aOR: 1.10, 95\% CI: 1.07, 1.12; $p<0.001$ ) were associated with higher odds of live discharge. 
Table 2. Multivariable logistic regression model for live discharge after ICPR.

\begin{tabular}{|c|c|c|}
\hline Variable & aOR $(95 \% \mathrm{CI})$ & $p$-Value \\
\hline Age & $0.98(0.98,0.99)$ & $<0.001$ \\
\hline Sex, male & $0.99(0.98,1.01)$ & 0.431 \\
\hline Have a job at ICPR & $1.05(1.03,1.07)$ & $<0.001$ \\
\hline \multicolumn{3}{|l|}{ Residence at ICPR } \\
\hline Urban area & 1 & \\
\hline Rural area & $0.91(0.90,0.93)$ & $<0.001$ \\
\hline \multicolumn{3}{|l|}{ Household income level at ICPR } \\
\hline Q1 & 1 & \\
\hline $\mathrm{Q} 2$ & $1.06(1.03,1.09)$ & $<0.001$ \\
\hline Q3 & $1.09(1.06,1.12)$ & $<0.001$ \\
\hline Q4 & $1.10(1.07,1.12)$ & $<0.001$ \\
\hline Unknown & $1.08(1.01,1.15)$ & 0.019 \\
\hline \multicolumn{3}{|l|}{ Main diagnosis at ICPR } \\
\hline Cardiovascular disease & 1 & \\
\hline Respiratory disease & $0.72(0.70,0.74)$ & $<0.001$ \\
\hline Cancer & $0.38(0.37,0.40)$ & $<0.001$ \\
\hline Other & $0.70(0.68,0.71)$ & $<0.001$ \\
\hline \multicolumn{3}{|l|}{ Admitting department } \\
\hline IM & 1 & \\
\hline Non-IM & $1.03(1.02,1.05)$ & $<0.001$ \\
\hline \multicolumn{3}{|l|}{ Duration of ICPR } \\
\hline$<15 \mathrm{~min}$ & 1 & \\
\hline $15-30$ & $0.42(0.41,0.43)$ & $<0.001$ \\
\hline $30-45$ & $0.29(0.28,0.30)$ & $<0.001$ \\
\hline $45-60$ & $0.26,0.25,0.27)$ & $<0.001$ \\
\hline$>60$ & $0.25(0.24,0.26)$ & $<0.001$ \\
\hline CCI, point & $1.03(1.02,1.03)$ & $<0.001$ \\
\hline \multicolumn{3}{|l|}{ Type of hospital } \\
\hline Tertiary general hospital & 1 & \\
\hline General hospital & $0.83(0.81,0.84)$ & $<0.001$ \\
\hline Other hospital & $1.06(1.02,1.09)$ & 0.001 \\
\hline \multicolumn{3}{|l|}{ Total hospital bed number } \\
\hline$<1000$ & 1 & \\
\hline$\geq 1000$ & $0.90(0.88,0.93)$ & $<0.001$ \\
\hline \multicolumn{3}{|l|}{ Year of ICPR } \\
\hline 2010 & 1 & \\
\hline 2011 & $1.00(0.96,1.05)$ & 0.921 \\
\hline 2012 & $1.01(0.97,1.056)$ & 0.605 \\
\hline 2013 & $0.89(0.85,0.93)$ & $<0.001$ \\
\hline 2014 & $0.90(0.86,0.94)$ & $<0.001$ \\
\hline 2015 & $0.91(0.87,0.95)$ & $<0.001$ \\
\hline 2016 & $0.92(0.89,0.96)$ & $<0.001$ \\
\hline 2017 & $0.89(0.86,0.93)$ & $<0.001$ \\
\hline 2018 & $0.85(0.81,0.88)$ & $<0.001$ \\
\hline 2019 & $0.88(0.85,0.92)$ & $<0.001$ \\
\hline
\end{tabular}

aOR, adjusted odds ratio; CI, confidence interval; ICPR, in-hospital cardiopulmonary resuscitation; IM, internal medicine; $\mathrm{CCI}$, Charlson comorbidity index.

\section{Discussion}

In this population-based cohort study in South Korea, the prevalence of ICPR increased from 2010 to 2019, but mortality rates remained high during this period: over $90 \%$ for 6-month and 1-year mortality after ICPR. Moreover, the financial burden of hospitalization at ICPR increased during the study period. As the main diagnosis at ICPR, cardiovascular increased, while cancer and respiratory disease decreased during the study period. We also show that there were many factors associated with live discharge after ICPR. This study reports important information regarding recent trends in ICPR using real-world data from the NHIS database of South Korea. 
In the United States, the prevalence of ICPR among elderly patients was reported as 2.73 events per 1000 admissions from 1992 to 2005 [8]. In South Korea, the prevalence of ICPR was 0.92 events per 1000 admissions in 2019, which was lower than that reported in a previous study [8]. The difference might be due to the inclusion of all adult patients in our study. For non-elderly patients in the United States, the prevalence of ICPR increased from 1.81 per 1000 admissions in 2007 to 2.37 per 1000 admissions in 2012 [5], suggesting that the prevalence of ICPR was relatively low in South Korea. Another cohort study in China reported that the prevalence of ICPR was 4.7 cases per 1000 admissions between 1 January and 31 December 2014, in 12 Beijing hospitals [9], and 1.6 cases per 1000 admissions from 1 April 2011 to 31 March 2013 in the United Kingdom [10]. Therefore, our study shows that the overall prevalence of ICPR among adult patients in South Korea was lower than that in other countries [5,8-10].

Our results demonstrated that the frequency of ICPR due to IHCA increased abruptly in 2016 and continuously increased until 2019 in South Korea. Some factors explain this jump. First, many hospitals have been built in South Korea as a result of national planning (https:/ / data.worldbank.org/indicator/SH.MED.BEDS.ZS?locations=KR, accessed on 7 September 2021), which could be associated with the increase in ICPR in South Korea. Second, the super-aged population in South Korea may increase hospitalization rates of elderly patients who were at higher risk of ICPR due to IHCA [11].

A previous meta-analysis in 1998 reported that the rate of survival to discharge after ICPR was $13.4 \%$ [12], which was lower than the one in our study (26.2\%). However, more recent findings indicate that the rate of survival to discharge after ICPR was $18.4 \%$ from 1 April 2011 to 31 March 2013 in the United Kingdom [10], and 34.0\% from 1 January 2015 to 31 December 2018 in the United States [13]. In addition to these findings [10,13], we showed that, in recent studies, rates of live discharge following ICPR were higher than those in previous studies [12]. The survival rates after ICPR were $9.8 \%$ at 6 months and $8.7 \%$ at 1 year in South Korea. A meta-analysis of 40 studies in 2018 reported that the pooled 1-year survival after ICPR following IHCA was 13.4\% [4], which was higher than that in our study. As shown in Figure 2, despite some advances in critical care, the mortality rates remain high at over $90 \%$ at 6 months and 1 year after ICPR in South Korea.

The financial burden for patients who received ICPR after IHCA increased from 2011 to 2019 in South Korea. The health care cost following ICPR also increased steadily in the United States [14]. In South Korea, the mean total cost of hospitalization at ICPR was USD 6753.50 (SD: USD 10,295.10), and approximately 90\% of the total cost was covered by the public health insurance program. This is because the NHIS in South Korea covers $95 \%$ of the total medical expenses for treatment and examinations for patients diagnosed with cancer, heart disease, cerebrovascular disease, rare intractable diseases, and severe burns [15].

As our study analyzed a large sample using a nationwide database, there were many interesting factors associated with live discharge after ICPR. As socioeconomic-statusrelated factors, employment status, urban residency, and higher household income level were associated in this study with higher odds of live discharge. A recent review reported that there was no clear relationship between socioeconomic status and outcomes after ICPR [16]. The impact of socioeconomic status on outcomes after ICPR might be influenced by various health care and social welfare systems in countries. Although most studies use insurance status to evaluate income level [16], we used national household income level, which is a strength of this study.

Interestingly, cancer, as the main diagnosis, was also associated with lower odds of live discharge after ICPR. In previous studies, the survival rate after ICPR for patients with cancer has been reported to be extremely poor $[17,18]$. A recent cohort study reported that, in oncology wards, $37.2 \%$ of patients received potentially avoidable CPR, which was defined as ICPR for patients who had no further chemotherapy plans, were in hospice care, or were expected to have worse clinical courses with irreversible prognosis [19]. In our 
cohort study, there may have been cases of potentially avoidable ICPR for patients with cancer, which may have affected the live discharge rate.

A longer ICPR duration was also associated with lower odds of live discharge. A retrospective single-center cohort study reported that the duration of ICPR was inversely associated with outcomes, and most of the benefits of ICPR could be achieved in the first $15 \mathrm{~min}$ [20]. In our study, $43.1 \%$ of patients received ICPR for $<15 \mathrm{~min}$, while ICPR duration over $30 \mathrm{~min}$ showed similar aORs as those for longer durations ( 0.29 in the 30-45 min group, 0.26 in the $45-60 \mathrm{~min}$ group, and 0.25 in the $>60$ min group) for live discharge.

This study has several limitations. First, we cannot extract data on important outcomes, such as the return of spontaneous circulation after ICPR, because of the lack of ICD-10 codes in the NHIS database. Second, the NHIS database lacks some important information, such as body mass index, alcohol consumption, and smoking status. Third, there may be residual and unmeasured confounders in our survival analysis of patients who received ICPR. Lastly, the results of our study might have limitations regarding generalizability because clinical practice of ICPR may be influenced by different cultures and health care systems in different countries.

\section{Conclusions}

In conclusion, from 2011 to 2019, there was an increase in the prevalence of ICPR and total costs of hospitalization at ICPR among adult patients in South Korea. The mortality rate remained high for ten years, and many factors, such as unemployment, lower household income level, older age, longer duration of ICPR, cancer as the main diagnosis, and urban residency, were associated with lower odds of live discharge after ICPR. This study provides information useful to hospitalized patients and their physicians in deciding whether to choose to be resuscitated individually considering the clinical benefit of ICPR and financial burden.

Supplementary Materials: The following supporting information can be downloaded at: https:// www.mdpi.com/article/10.3390/jpm12030377/s1, Table S1. The ICD-10 codes used by comorbidity to compute the Charlson comorbidity index, Table S2. Prevalence of ICPR in South Korea among the adult population from 2010 to 2019, Table S3. Mortality rates after ICPR from 2010 to 2019, Table S4. Mean value of total cost of hospitalization at ICPR from 2010 to 2019 in USD, Table S5. Trends in the main diagnosis at ICPR from 2010 to 2019, Table S6. Trends in the duration of ICPR from 2010 to 2019, Figure S1. Trends in the main diagnosis at ICPR from 2010 to 2019, Figure S2. Trends in the duration of ICPR from 2010 to 2019, Figure S3. Flow chart depicting study population selection process.

Author Contributions: Conceptualization, T.K.O. and I.-A.S.; methodology, T.K.O.; software, T.K.O.; formal analysis, I.-A.S.; investigation, Y.H.J.; data curation, T.K.O.; writing—original draft preparation, T.K.O., I.-A.S. and Y.H.J.; writing-review and editing. All authors have read and agreed to the published version of the manuscript.

Funding: This research received no external funding.

Institutional Review Board Statement: The study protocol was approved by the institutional review board (IRB; X-2011-651-901), and the National Health Insurance Service (NHIS) permitted data sharing after approval of the study protocol (NHIS-2021-1-266).

Informed Consent Statement: The requirement for informed consent was waived by the IRB because anonymized data were used in this study.

Data Availability Statement: All data will be available upon reasonable request to corresponding author.

Conflicts of Interest: The authors declare no conflict of interest. 


\section{References}

1. Andersen, L.W.; Holmberg, M.J.; Berg, K.M.; Donnino, M.W.; Granfeldt, A. In-Hospital Cardiac Arrest: A Review. JAMA 2019, 321, 1200-1210. [CrossRef] [PubMed]

2. Girotra, S.; Nallamothu, B.K.; Spertus, J.A.; Li, Y.; Krumholz, H.M.; Chan, P.S.; Yan American Heart Association Get with the Guidelines-Resuscitation Investigators. Trends in survival after in-hospital cardiac arrest. N. Engl. J. Med. 2012, 367, 1912-1920. [CrossRef]

3. Choi, Y.; Kwon, I.H.; Jeong, J.; Chung, J.; Roh, Y. Incidence of Adult In-Hospital Cardiac Arrest Using National Representative Patient Sample in Korea. Healthc. Inform. Res. 2016, 22, 277-284. [CrossRef] [PubMed]

4. Schluep, M.; Gravesteijn, B.Y.; Stolker, R.J.; Endeman, H.; Hoeks, S.E. One-year survival after in-hospital cardiac arrest: A systematic review and meta-analysis. Resuscitation 2018, 132, 90-100. [CrossRef] [PubMed]

5. Mallikethi-Reddy, S.; Briasoulis, A.; Akintoye, E.; Jagadeesh, K.; Brook, R.D.; Rubenfire, M.; Afonso, L.; Grines, C.L. Incidence and Survival After In-Hospital Cardiopulmonary Resuscitation in Nonelderly Adults: US Experience, 2007 to 2012. Circ. Cardiovasc. Qual. Outcomes 2017, 10, 2. [CrossRef] [PubMed]

6. $\quad$ Park, I.Y.; Ju, Y.S.; Lee, S.Y.; Cho, H.S.; Hong, J.I.; Kim, H.A. Survival after in-hospital cardiopulmonary resuscitation from 2003 to 2013: An observational study before legislation on the life-sustaining treatment decision-making act of Korean patients. Medicine 2020, 99, e21274. [CrossRef] [PubMed]

7. Vandenbroucke, J.P.; Von Elm, E.; Altman, D.G.; Gøtzsche, P.C.; Mulrow, C.D.; Pocock, S.J.; Poole, C.; Schlesselman, J.J.; Egger, M.; Initiative, S. Strengthening the Reporting of Observational Studies in Epidemiology (STROBE): Explanation and elaboration. PLoS Med. 2007, 4, e297. [CrossRef] [PubMed]

8. Ehlenbach, W.J.; Barnato, A.E.; Curtis, J.R.; Kreuter, W.; Koepsell, T.D.; Deyo, R.A.; Stapleton, R.D. Epidemiologic study of in-hospital cardiopulmonary resuscitation in the elderly. N. Engl. J. Med. 2009, 361, 22-31. [CrossRef] [PubMed]

9. Shao, F.; Li, C.S.; Liang, L.R.; Qin, J.; Ding, N.; Fu, Y.; Yang, K.; Zhang, G.Q.; Zhao, L.; Zhao, B.; et al. Incidence and outcome of adult in-hospital cardiac arrest in Beijing, China. Resuscitation 2016, 102, 51-56. [CrossRef]

10. Nolan, J.P.; Soar, J.; Smith, G.B.; Gwinnutt, C.; Parrott, F.; Power, S.; Harrison, D.A.; Nixon, E.; Rowan, K.; National Cardiac Arrest, A. Incidence and outcome of in-hospital cardiac arrest in the United Kingdom National Cardiac Arrest Audit. Resuscitation 2014, 85, 987-992. [CrossRef] [PubMed]

11. Kim, K.W.; Kim, O.S. Super aging in South Korea unstoppable but mitigatable: A sub-national scale population projection for best policy planning. Spat. Demogr. 2020, 8, 155-173. [CrossRef] [PubMed]

12. Ebell, M.H.; Becker, L.A.; Barry, H.C.; Hagen, M. Survival after in-hospital cardiopulmonary resuscitation. A meta-analysis. J Gen. Intern. Med. 1998, 13, 805-816. [CrossRef] [PubMed]

13. Girotra, S.; Nallamothu, B.K.; Tang, Y.; Chan, P.S.; American Heart Association Get With The Guidelines-Resuscitation Investigators. Association of Hospital-Level Acute Resuscitation and Postresuscitation Survival With Overall Risk-Standardized Survival to Discharge for In-Hospital Cardiac Arrest. JAMA Netw. Open 2020, 3, e2010403. [CrossRef] [PubMed]

14. Damluji, A.A.; Al-Damluji, M.S.; Pomenti, S.; Zhang, T.J.; Cohen, M.G.; Mitrani, R.D.; Moscucci, M.; Myerburg, R.J. Health Care Costs After Cardiac Arrest in the United States. Circ. Arrhythmia Electrophysiol. 2018, 11, e005689. [CrossRef] [PubMed]

15. Kwon, S. Thirty years of national health insurance in South Korea: Lessons for achieving universal health care coverage. Health Policy Plan. 2009, 24, 63-71. [CrossRef] [PubMed]

16. Stankovic, N.; Hoybye, M.; Lind, P.C.; Holmberg, M.; Andersen, L.W. Socioeconomic status and in-hospital cardiac arrest: A systematic review. Resusc. Plus 2020, 3, 100016. [CrossRef] [PubMed]

17. Lee, M.R.; Yu, K.L.; Kuo, H.Y.; Liu, T.H.; Ko, J.C.; Tsai, J.S.; Wang, J.Y. Outcome of stage IV cancer patients receiving in-hospital cardiopulmonary resuscitation: A population-based cohort study. Sci. Rep. 2019, 9, 9478. [CrossRef] [PubMed]

18. Varon, J.; Walsh, G.L.; Marik, P.E.; Fromm, R.E. Should a cancer patient be resuscitated following an in-hospital cardiac arrest? Resuscitation 1998, 36, 165-168. [CrossRef]

19. Choi, Y.; Kim, J.W.; Suh, K.J.; Lim, Y.J.; Lee, J.Y.; Kang, B.D.; Kim, J.W.; Kim, S.H.; Lee, J.O.; Kim, Y.J.; et al. Identification of a potentially avoidable cardiopulmonary resuscitation in hematology and oncology wards. BMC Palliat. Care 2019, 18, 93. [CrossRef] [PubMed]

20. Cheema, M.A.; Ullah, W.; Abdullah, H.M.A.; Haq, S.; Ahmad, A.; Balaratna, A. Duration of in-hospital cardiopulmonary resuscitation and its effect on survival. Indian Heart J. 2019, 71, 314-319. [CrossRef] 